\title{
Viajar con niños. Lo que debe saber el pediatra
} Travelling with children

\author{
Dra. María Laura Praino ${ }^{a}$, Dra. Griselda Berberián ${ }^{b}$, Dra. Cecilia Torroijac, \\ Comisión de Pediatría de la Sociedad Latinoamericana de Medicina del Viajero \\ (SLAMVI)
}

\begin{abstract}
RESUMEN
Cada vez resulta más frecuente que los niños realicen viajes a sitios muy diferentes de su lugar de residencia. Independientemente de cuál sea su motivo, implica una exposición a un medioambiente con características propias que deben ser tenidas en cuenta. De igual manera, el traslado en sí mismo puede generar ansiedad en la familia y presenta sus propios riesgos. Es frecuente que los padres realicen una consulta con un profesional, habitualmente su pediatra de cabecera, durante la planificación de un viaje. El objetivo del presente trabajo es revisar las recomendaciones para el asesoramiento previo. Inicialmente, se abordarán recomendaciones generales en la preparación del viaje y luego la prevención de enfermedades transmitidas por insectos.

Palabras clave: niños, viajes, prevención.
\end{abstract}

\section{ABSTRACT}

The number of children who travel outside their home countries has increased dramatically. Whatever the reason for it, it involves exposure to an environment with characteristics that must be taken into account. Similarly, the transfer itself can generate anxiety in the family and presents the risk of vehicle-related injuries (which is the leading cause of death in children who travel). Parents will often seek for pre-travel advice. The aim of this paper is to review the recommendations to assess a family who is planning to travel with children. Initially, general recommendations will be addressed in the preparation of the trip and afterwards insectborne diseases prevention.

Key words: travel, children, prevention.

http:/ /dx.doi.org/10.5546/aap.2015.58

\section{INTRODUCCIÓN}

De acuerdo con la Organización Mundial de Turismo de las Naciones Unidas, se estima que, en el año 2012, el número de personas que viajan por el mundo ha llegado a 1035 millones. ${ }^{1}$ El número de niños que realizan viajes internacionales también ha ido aumentando en proporción, aunque existen pocos datos. En 2010, se calculaba que aproximadamente
2,2 millones de niños residentes en los Estados Unidos habían realizado viajes internacionales. ${ }^{2}$ Más de la mitad de los viajes son realizados por motivos turísticos (51\%), un $15 \%$ por motivos laborales y otro $27 \%$ por causas específicas, entre las que se encuentran quienes visitan a familiares y amigos. ${ }^{3}$ Este último grupo representa un riesgo particular. Esto se debe a que tienden a adoptar los hábitos de quienes visitan sin tener en cuenta que los riesgos pueden ser más importantes para ellos. Por ejemplo, estar habituados a vivir en la altura o poseer inmunidad parcial frente a la malaria.

Viajar con niños puede ser una hermosa experiencia, interesante para toda la familia, pero presenta desafíos particulares que deben ser tenidos en cuenta al momento de organizar un viaje, con el fin de evitar las complicaciones.

El viaje se divide en tres períodos, que son el previaje, el viaje propiamente dicho y el posviaje; cada uno requiere consideraciones particulares.

El objetivo del presente trabajo es actualizar las recomendaciones para viajeros en edad pediátrica, de manera que los profesionales actuantes puedan asesorar a las familias en su consulta previa al viaje.

La presentación está basada en estos temas iniciales:

\section{- Recomendaciones generales}

a. Traslado.

b. Prevención de accidentes.

c. Exposición solar.

d. Riesgos en la altura.

- Enfermedades transmitidas por insectos 


\section{Recomendaciones generales}

La preparación del viaje puede ser una situación estresante para los niños, en especial, cerca del momento de la partida. Los padres deben tenerlo en cuenta para planificar todo con la mayor anticipación posible, considerando la probabilidad de que haya demoras.

Incluir a los niños en la preparación del viaje disminuye la ansiedad o temor frente a este. Entre las cosas para llevar, se recomiendan objetos que les sean familiares, como muñecos, juguetes, mantita, etc.

Se aconseja fuertemente contratar seguro de viajes ya que se calcula que casi las dos terceras partes de los niños requerirán algún tipo de atención médica.

\section{EL TRASLADO \\ En avión}

Si bien se recomienda el uso de butacas para niños similares a las usadas para autos, esto no es así en la práctica. No todas las butacas se adaptan a los asientos de los aviones y los menores de 2 años suelen viajar en brazos de sus padres para disminuir los costos.

A pesar de que las cabinas de los aviones comerciales están presurizadas, la presión inspirada de oxígeno durante el vuelo es menor a la que se respira a nivel del mar, lo que podría causar hipoxemia. Se recomienda esperar, al menos, a la semana de vida (en los nacidos a término) para realizar el viaje o a las 40 semanas de edad postconcepcional (para los prematuros sin patología pulmonar). Las recomendaciones para quienes padecen patología pulmonar deben ser individualizadas ya que algunos pacientes podrían requerir oxígeno suplementario durante el vuelo. ${ }^{4,5}$

Puede presentarse dolor de oídos por el cambio de presiones, en especial al momento de la partida y del aterrizaje. Para disminuir dicha molestia, se recomienda mascar chicle o succionar (chupete o mamadera), de acuerdo con la edad. No está demostrado que el tratamiento farmacológico sea de utilidad.

\section{"Jet lag"}

Al cambio de uso horario y sus consecuencias sobre el ritmo circadiano se les da el nombre de "jet lag". Se encuentran frente a un mayor riesgo quienes realizan viajes en avión que cruzan más de 3 husos horarios. Se recomiendan las siguientes medidas generales para prevenir o acortar la duración de los síntomas: adecuado descanso previo, consumir una dieta saludable los días anteriores al viaje, adaptar paulatinamente el horario al destino (ej., acostarse $2 \mathrm{~h}$ antes si se viajará hacia el este) los 2-3 días previos y acomodar lo antes posible el horario de sueño y comidas al llegar a destino. ${ }^{6}$ No se recomienda el uso de melatonina, especialmente en adolescentes, en quienes podría afectar la maduración sexual (aumento de prolactina y disminución de testosterona).

En cuanto a la sedación para el viaje, las benzodiacepinas están contraindicadas. Una alternativa es la difenhidramina. Dado que esta droga conlleva el riesgo de excitación paradojal, sobre todo en menores de 2 años, es importante que los padres prueben, antes del viaje, el efecto de esta droga en sus hijos.

En casos de cinetosis, el dimenhidrato puede indicarse con cautela. No se recomienda el uso de metoclopramida debido al riesgo de efectos adversos extrapiramidales.

\section{En auto}

Los accidentes en vehículos a motor son la principal causa de muerte en niños que viajan. Deben tenerse en cuenta las siguientes recomendaciones: ${ }^{78}$

- Los niños hasta los 12 años deben viajar en los asientos de atrás.

- Los lactantes deben viajar mirando hacia atrás hasta tener 2 años o hasta que hayan superado el límite de talla o peso de la silla para lactantes.

- A partir de los 2 años y hasta los 4 años, se recomiendan las sillas en posición frontal y con arnés.

- Los niños están más seguros en las sillas para niños mientras entren.

- Los niños mayores pueden usar sillas para adaptar el uso del cinturón de seguridad del auto de manera que este apoye sobre estructuras óseas fuertes (hombro-cadera). Este tipo de sillas, llamadas "booster", pueden ser con o sin respaldo. Las primeras ayudan a adaptar la posición del cinturón de seguridad de 3 puntos.

- Las "airbags" frontales pueden ser peligrosas para niños con talla menor de 1,50 metros, por lo que no deben ir sentados en asientos delanteros.

- En la cabina del automóvil, no deben llevarse objetos sueltos (juguetes, lápices, botellas, etc.) ya que, si se produjera un accidente, su impacto podría provocar graves lesiones. 


\section{PREVENCIÓN DE ACCIDENTES}

Las medidas de prevención de accidentes son similares a las recomendadas en forma habitual. Se sugiere la lectura del Manual de prevención de accidentes de la Sociedad Argentina de Pediatría. ${ }^{9}$ En situación de viaje, el cambio de ambiente y rutina aumenta el riesgo.

Solo se hará mención a los accidentes con agua ya que son la segunda causa de muerte en viajeros, especialmente en menores de 5 años.,10,11 Es importante que, si la familia va a hospedarse en un lugar con piscinas, estas cuenten con un cerco de protección. Además, en cercanías de piscinas, ríos, lagos o costas de mar, los niños pequeños deben estar siempre al cuidado de un adulto (no de hermanos mayores), aun aquellos que saben flotar.

\section{EXPOSICIÓN SOLAR}

La radiación solar puede ser dañina. Los niños se encuentran en mayor riesgo que los adultos de padecer complicaciones, tales como golpe de calor, eritema, cáncer de piel y fototoxicidad. . $^{12,13}$

El riesgo es mayor en regiones próximas al ecuador, en verano, en horas cercanas al mediodía (de 10 a.m. a 4 p.m.) y, en especial, al desarrollar actividades en lugares que reflejen la luz solar, tales como arena, agua o nieve. De cualquier modo, no debe desestimarse el riesgo por exposición indirecta al sol (bajo una sombrilla o en días nublados), especialmente en el caso de lactantes.

Además, debe tenerse en cuenta que algunos medicamentos pueden producir fotosensibilidad (entre ellos, antibióticos con las sulfamidas y las tetraciclinas). Ciertas enfermedades aumentan el riesgo de complicaciones, tales como las enfermedades del tejido conectivo, rosácea, vitiligo, entre otras.

Las medidas de protección incluyen las siguientes:

- Evitar estar al sol en horarios cercanos al mediodía.

- Evitar hacer actividad física intensa cuando la temperatura es elevada.

- Tener en cuenta que pueden producirse quemaduras aun en días nublados o en la sombra.

- Usar ropa adecuada: colores oscuros, trama cerrada o indumentaria especialmente diseñada para este fin (que aclara el índice de protección solar) y usar sombreros.

- Usar lentes para sol con filtros UV si se realizarán actividades en zonas que reflejen la luz solar, por ejemplo, en la nieve.
- Ofrecer agua con frecuencia ya que los niños pueden no solicitarla hasta estar deshidratados.

- Usar cremas con protección solar UVA y UVB. Idealmente, deben elegirse aquellos productos que especifiquen su fecha de vencimiento ya que pierden eficacia con el tiempo. El riesgo de dermatitis de contacto con estos productos es proporcional al factor de protección solar (FPS), por lo que se sugiere el uso de productos con un FPS de, al menos, 15 o 30. El protector solar debe ser recolocado cada $2 \mathrm{~h}$ y luego de mojarse. Se debe tener en cuenta que el uso de repelentes de mosquito se realiza sobre el protector solar y disminuye su eficacia, por lo que deben elegirse cremas con índices de protección más altos. No se recomiendan los productos que combinan repelente y protector solar ya que este último requiere ser aplicado con mayor frecuencia que el repelente.

- No exponerse a camas solares.

Los lactantes de menos de 6 meses no deben ser expuestos en forma directa al sol. Si la exposición es inevitable, deben usar ropa que cubra la mayor parte del cuerpo y sombrero, y pueden usar protector solar únicamente en las zonas expuestas teniendo en cuenta que la seguridad de los productos en este grupo etario no está aclarada.

Frente a quemaduras solares, se sugiere ofrecer abundantes líquidos frescos al niño de manera de lograr una adecuada hidratación. La colocación de cremas o geles hidratantes mejora síntomas locales. Dado que las quemaduras pueden producir síntomas sistémicos, como fiebre o malestar general, se recomienda el uso de antiinflamatorios no esteroides. También es apropiado el uso de antihistamínicos, como la difenhidramina para disminuir el prurito.

\section{RIESGOS EN LA ALTURA}

Si bien las características generales del clima en la altura exponen a diferentes patologías (menor humedad, mayor exposición a radiación ultravioleta, temperaturas más frías), la mayor preocupación es el "mal de altura". Si bien la susceptibilidad al mal de altura es variable, el riesgo es similar en niños y en adultos. Se presenta por la exposición a regiones que se encuentran a más de $2500 \mathrm{msnm}$ (metros sobre el nivel del mar) y puede llegar a comprometer a más de un tercio de los niños durante el primer día en ascensos rápidos. ${ }^{14}$

El mal de altura se manifiesta con cefalea, 
síntomas gastrointestinales (anorexia, náuseas y vómitos), insomnio, fatiga, mareos. En grados más avanzados, aparece dificultad respiratoria secundaria a edema pulmonar y síntomas neurológicos, como ataxia y alteración de la conciencia.

Los factores determinantes del riesgo son los siguientes:

- Velocidad de ascenso.

- Altura final.

- Altura a la que se duerme.

- Factores individuales (antecedente de mal de altura, vivir a menos de $900 \mathrm{~m}$, realizar ejercicio al llegar, patología preexistente cardiopulmonar).

La forma de evitar los síntomas del mal de altura es realizar un ascenso gradual (no subir más de 500 metros por día). Puede usarse medicación como profilaxis de mal de altura cuando el ascenso rápido es inevitable (acetazolamida), pero parecería no ser necesaria en niños. En caso de aparecer síntomas, se debe discontinuar el ascenso. Si está disponible, se debe ofrecer oxígeno suplementario a 2 litros/minuto. El tratamiento farmacológico incluye el uso de analgésicos, antieméticos y dexametasona. Si los síntomas persisten, es obligatorio descender, al menos, 300 metros. Habitualmente, los síntomas revierten, pero en algunos casos, es necesario el uso de oxígeno suplementario o, incluso, cámara hiperbárica en situaciones graves. ${ }^{15}$

\section{ENFERMEDADES TRANSMITIDAS POR INSECTOS}

Las enfermedades transmitidas por insectos son especialmente frecuentes en regiones tropicales. Si bien para algunas de ellas existe una prevención específica, para muchas otras no la hay. Por este motivo, se debe poner énfasis en evitar las picaduras de mosquitos, otros insectos $\mathrm{u}$ artrópodos.

Según una publicación reciente, para 2010, se estimaba que los casos de dengue anuales serían de 390 millones a nivel mundial, aunque solo 96 millones serían clínicamente aparentes. ${ }^{16}$

Las picaduras de mosquito, aparte de ser molestas, pueden ocasionar reacciones alérgicas o urticariformes, especialmente en la cara. Además, conllevan el riesgo de sobreinfección bacteriana $y$, muchas veces, son una puerta de entrada para la formación de celulitis. Las picaduras de mosquitos y artrópodos representan una importante causa de consulta en el posviaje. ${ }^{17}$

Debe tenerse en cuenta el horario de mayor riesgo de picaduras de mosquitos (el vector de dengue o chikungunya pica más durante el día, mientras que el de malaria lo hace especialmente, durante el amanecer o el atardecer).

Para evitar picaduras de insectos, se sugiere el uso de ropa de manga larga y trama cerrada, pantalones largos y calzado cerrado. Algunos productos, como la permetrina, pueden ser aplicados a los mosquiteros o la ropa, lo que aumenta la eficacia contra los mosquitos. Al sentarse en el suelo, el uso de mantas ayudará a prevenir lesiones por artrópodos en la arena o el pasto. ${ }^{18}$

Las habitaciones deben contar con mosquiteros en buen estado, que también se pueden colocar sobre cunas o cochecitos de paseo.

Se pueden usar insecticidas ambientales evitando la inhalación directa del humo o spray de estos.

Los repelentes para mosquitos de uso individual tienen una eficacia variable. Los de mayor eficiencia son los que contienen $\mathrm{N}, \mathrm{N}$ dietil-meta-toluamida (DEET). Se presentan en concentraciones variables, que van del 6 al $30 \%$, y se prolonga el tiempo de eficacia del producto en concentraciones más altas. ${ }^{19} \mathrm{No}$ deben ser colocados a menores de dos meses de vida. Se debe ser cauto en su uso ya que pueden absorberse a través de la piel, por lo que se sugiere colocarlos solo en superficies expuestas, en los períodos en que se estará al aire libre y lavar con agua y jabón al regresar para quitar el excedente. Si el producto por usar es un aerosol, no debe ser aplicado en ambientes cerrados donde se encuentran niños pequeños.

Existen otros tipos de repelentes con componentes tales como la citronela. Su eficacia es notablemente peor que los que usan DEET, especialmente los que tienen bajas concentraciones del fármaco, por lo que no se recomiendan.

\section{Otras enfermedades infectocontagiosas}

El pediatra debe controlar el carnet de inmunizaciones con antelación suficiente para poder indicar nuevas vacunas o dosis ulteriores, si fuera necesario. La mayoría de los destinos no requieren el uso de agentes diferentes de los que se indican en el programa nacional de inmunizaciones.

Las enfermedades transmitidas por agua $y$ alimentos son relativamente frecuentes en viajeros. El tema no será abordado en esta revisión debido a que requiere un análisis más extenso y particular. 


\section{EL BOTIQUÍN}

Es frecuente que los padres le consulten al pediatra qué deberían llevar en un botiquín para el viaje. A continuación, se listan algunos productos de uso frecuente, que deberán ser adecuados según cada situación en particular.

- Alcohol en gel.

- Protector solar.

- Repelentes contra insectos a base de DEET.

- Solución desinfectante con iodopovidona.

- Sales de rehidratación oral.

- Cremas: humectante, con corticoides y con antimicóticos.

- Antitérmicos y analgésicos: paracetamol, ibuprofeno o dipirona.

- Antihistamínicos, como la difenhidramina.

De acuerdo con las características particulares de la familia y del viaje, pueden ser necesarios otros medicamentos o sus recetas (con nombre genérico).

\section{CONCLUSIONES}

El número de niños que realizan viajes internacionales ha ido en aumento en los últimos años. Para que el viaje pueda ser una agradable experiencia para toda la familia, es necesario realizar una adecuada preparación.

Se sugiere incluir al niño en la preparación del viaje. Deben tenerse en cuenta las características particulares del traslado a cada edad para evitar complicaciones o posibles accidentes.

La protección solar es fundamental, al igual que las medidas de prevención de picaduras de insectos.

El objetivo de la consulta previaje será prevenir todo lo que sea posible $y$, sobre todo, anticipar situaciones adversas que puedan aparecer durante el viaje. De esta manera, la familia podrá abordarlas con tranquilidad, sabiendo cuál es la mejor conducta para tomar y cuándo es necesario realizar una consulta médica.

\section{REFERENCIAS}

1. World Tourism Organization. International tourism expected to see robust growth in 2013. UNWTO World Tourism Barometer 2013;11:1-2.

2. Weinberg $\mathrm{N}$, Weinberg M, Maloney SA. International travel with infants and children. En: Centers for Disease Control and Prevention, ed. CDC Health information for international travel 2012. New York: Oxford University Press; 2012. Págs.490-6.

3. Organizzazione Mondiale della Sanità. Società Italiana di Medicina del Turismo. Viaggi internazionali e salute: Situazione al 1 gennaio 2012: Rischi per la salute e precauzioni da prendere: generalità . Rimini, 2012. [Disponible en: http://apps.who.int/iris/ bitstream/10665/75329/1/9789241580472_ita.pdf?ua=1]. [Acceso: 22 de agosto de 2014].

4. Aguerre V. Viaje en avión, riesgos y previsiones a considerar en los niños. Arch Argent Pediatr 2012;110(1):66-9.

5. British Thoracic Society Standards of Care Committee. Managing passengers with respiratory disease planning air travel: British Thoracic Society recommendations. Thorax 2002;57(4):289-304.

6. Sack RL. Clinical practice. Jet lag. $N$ Engl J Med 2010;362(5):440-7.

7. Navarro LD. ¿Qué deben saber los pediatras sobre las sillitas para autos? Sistemas de retención infantil para automóviles. Arch Argent Pediatr 2013;111(3):247-50.

8. Bull MJ, Sheese J. Update for the pediatrician on child passenger safety: five principles for safer travel. Pediatrics 2000;106(5):1113-6.

9. Subcomisión de Prevención de Accidentes. Manual de Prevención de Accidentes. Buenos Aires: Sociedad Argentina de Pediatría; 2001.Págs.46-63.

10. Cortés LM, Hargarten SW,Hennes HM. Recommendations for water safety and drowning prevention for travelers. J Travel Med 2006;13(1):21-34.

11. Subcomisión de Prevención de Accidentes. Consenso Nacional de Prevención del ahogamiento. "El niño y el agua". 1 ${ }^{a}$ parte. Arch Argent Pediatr 2009;107(3):271-6.

12. Ultraviolet light: a hazard to children. American Academy of Pediatrics. Committee on Environmental Health. Pediatrics 1999;104(2 Pt 1):328-33.

13. Climatic heat stress and the exercising child and adolescent. American Academy of Pediatrics. Committee on Sports Medicine and Fitness. Pediatrics 2000;106(1 Pt 1):158-9.

14. Bloch J, Duplain H, Rimoldi SF, Stuber T, et al. Prevalence and time course of acute mountain sickness in older children and adolescents after rapid ascent to 3450 meters. Pediatrics 2009;123(1):1-5.

15. Hackett PH, Shlim DR. Altitude illness. En Centers for Disease Control and Prevention, ed.CDC Health information for international travel 2012. New York: Oxford University Press; 2012. Págs. 60-5.

16. Bhatt S, Gething PW, Brady OJ, Messina JP, et al. The global distribution and burden of dengue. Nature 2013;496(7446):504-7.

17. Lederman ER, Weld LH, Elyazar IR, von Sonnenburg F, et al. Dermatologic conditions of the ill returned traveler: an analysis from the GeoSentinel Surveillance Network. Int J Infect Dis 2008;12(6):593-602.

18. Stauffer WM, Kamat D, Magill AJ. Travelling with infants and children. Part IV: insect avoidance and malaria prevention. J Travel Med 2003;10(4):225-40.

19. Fradin MS, DayJF. Comparative efficacy of insect repellents against mosquito bites. $N$ Engl J Med 2002;347(1):13-8. 